\title{
Automated Quantification of Macular Vasculature Changes from OCTA Images of Hematologic Patients
}

Engberg, Astrid Margareta Elisabet; Amini, Abdullah; Willerslev, Anne; Larsen, Michael; Sander, Birgit; Kessel, Line; Dahl, Anders Bjørholm; Dahl, Vedrana Andersen

\section{Published in:}

Proceedings of 17 th IEEE International Symposium on Biomedical Imaging

Link to article, DOI:

10.1109/ISBI45749.2020.9098441

Publication date:

2020

Document Version

Peer reviewed version

Link back to DTU Orbit

Citation (APA):

Engberg, A. M. E., Amini, A., Willerslev, A., Larsen, M., Sander, B., Kessel, L., Dahl, A. B., \& Dahl, V. A. (2020). Automated Quantification of Macular Vasculature Changes from OCTA Images of Hematologic Patients. In Proceedings of 17th IEEE International Symposium on Biomedical Imaging (pp. 1987-1991). [9098441] IEEE. https://doi.org/10.1109/ISBI45749.2020.9098441

\section{General rights}

Copyright and moral rights for the publications made accessible in the public portal are retained by the authors and/or other copyright owners and it is a condition of accessing publications that users recognise and abide by the legal requirements associated with these rights.

- Users may download and print one copy of any publication from the public portal for the purpose of private study or research.

- You may not further distribute the material or use it for any profit-making activity or commercial gain

- You may freely distribute the URL identifying the publication in the public portal 


\title{
AUTOMATED QUANTIFICATION OF MACULAR VASCULATURE CHANGES FROM OCTA IMAGES OF HEMATOLOGIC PATIENTS
}

\author{
Astrid M. E. Engberg ${ }^{1} \quad$ Abdullah Amini ${ }^{2,3} \quad$ Anne Willerslev ${ }^{2,3} \quad$ Michael Larsen $^{2,3}$ \\ Birgit Sander $^{2} \quad$ Line Kessel ${ }^{2,3} \quad$ Anders B. Dahl ${ }^{1} \quad$ Vedrana A. Dahl $^{1}$ \\ ${ }^{1}$ Department of Applied Mathematics and Computer Science, Technical University of Denmark \\ 2 The Eye Clinic, Rigshospitalet-Glostrup, Denmark \\ ${ }^{3}$ Department of Clinical Medicine, Faculty of Health and Medical Sciences, University of Copenhagen
}

\begin{abstract}
Abnormal blood compositions can lead to abnormal blood flow which can influence the macular vasculature. Optical coherence tomography angiography (OCTA) makes it possible to study the macular vasculature and potential vascular abnormalities induced by hematological disorders. Here, we investigate vascular changes in control subjects and in hematologic patients before and after treatment. Since these changes are small, they are difficult to notice in the OCTA images. To quantify vascular changes, we propose a method for combined capillary registration, dictionary-based segmentation and local density estimation. Using this method, we investigate three patients and five controls, and our results show that we can detect small changes in the vasculature in patients with large changes in blood composition.
\end{abstract}

Index Terms- OCTA, Quantification, Microvasculature

\section{INTRODUCTION}

Chronic myeloid leukemia, essential thrombocytosis and polycythemia vera belong to a category of chronic myeloid malignancies, which are characterized by abnormally high concentrations of either leukocytes, thrombocytes or red blood cells. Visual disturbances and headaches may be present due to the impaired blood flow [1].

The eye makes it possible to study how the microvasculature is affected. Abnormal blood composition has been shown to lead to abnormalities of structural optical coherence tomography (OCT) patterns inside retinal arteries and veins, and inspection of motion-contrast images has shown that hyperviscosity can be associated with prominent dilation of the retinal capillaries [1]. However, the details of what goes on inside the retinal capillaries cannot be resolved by OCT at present.

Optical coherence tomography angiography (OCTA) is a relatively new and non-invasive imaging modality used to capture the retinal microvasculature. In this work, we investigate whether OCTA scans can be used to quantify vascular changes in some hematologic disorders. We extract local capillary densities from OCTA data and compare these densities between two scans of the same person before and after treatment. We demonstrate the feasibility of our method by applying it to a small control group and to hematologic patients, where we know how the blood composition changes after treatment.

\subsection{Related work}

One of the main challenges when working with OCTA images is the complexity of the anatomical structures exhibiting subtle changes in intensities combined with the high acquisition noise. Several quantification schemes for extracting vascular features from OCTA images have been proposed. This includes algorithms based on thresholding [2, 3, 4] and more advanced methods like local fractal dimension or probabilistic modeling [5, 6], while some OCTA platforms provide their own quantification software $[7,8,9]$.

In this work, we investigate changes induced by treatment. Related to this, Dongye et al. [10] propose an algorithm to detect dilated capillaries in OCTA based on contrast and saliency methods. However, as mentioned in their paper, the algorithm is not developed for angiograms where most vessels are dilated. Hence, it may not be suitable for patients with hematological disorders, where the whole microvasculature is believed to be affected. Hagag et al. [11] investigate vascular changes in response to hyperoxia, based on flow index and vessel density. Other studies also look at temporal changes in the vessel density [12, 13]. Jeffrey et al. [14] show that vessel density decreases with signal strength. Therefore, temporal comparisons should be done with care.

\section{PROPOSED APPROACH}

We propose an approach to measure microvascular changes in the parafoveal region, based on a pair of en face angiograms each consisting of the superficial retinal layer (SRL) and the deep retinal layer (DRL). First, we use a dictionary-based model to identify the larger vessels and the capillaries in the 
scans. Second, we align the scans from different imaging sessions and then locally compare the capillary density.

\subsection{Dictionary-based segmentation}

To detect larger vessels (arterioles and venules), background and capillaries we are using a dictionary-based segmentation approach proposed in [15]. Here, it is assumed that the appearance of the vascular network can be represented as a combination of characteristic image features. The approach is described in detail in [16]. We use the same parameters and dictionary as in [15], although we here apply an initial adaptive histogram equalization to improve the contrast of the images. The DRL consists only of capillaries, so we combine the detected capillaries and larger vessels in this layer into one class. Examples of the data and the output can be seen in Fig. 1.

\subsection{Local density}

The local capillary density is a unit-less ratio computed in a circular kernel with a radius of 100 pixels or $0.3 \mathrm{~mm}$. This kernel is large enough to smooth out motion and acquisition artefacts, while small enough to still show local areas of interest. The effect of larger vessels is removed by computing the density as the amount of pixels belonging to the capillary class divided by the amount of pixels belonging to either background or capillaries in that area. Additionally, the single score global capillary density is calculated in an annulus with radius $0.5 \mathrm{~mm}$ and $1.3 \mathrm{~mm}$ surrounding the foveal center.

\subsection{Registration}

For a more precise measure of changes in the vascular network, we align the OCTA scans of the same person. We first apply a landmark-based registration on the SRL from the two scans. The landmarks are computed as Scale-Invariant Feature Transform (SIFT) features using the VLFeat library $[17,18]$. The SIFT descriptors between the scans are matched using Euclidean distance, and outliers are excluded by applying random sample consensus. Afterwards, an affine registration is applied using a mean squared difference similarity metric and a gradient descent optimizer. The estimated transformation is also applied to the DRL.

\subsection{Local comparison}

We can now compare the local capillary density of two different scans of the same eye. Local density change, with values between -1 and 1 , is computed by subtracting two local density images.

\section{EXPERIMENTAL SETUP}

Our aim is to establish whether our method detects changes in the microvasculature. We apply the method to healthy sub-
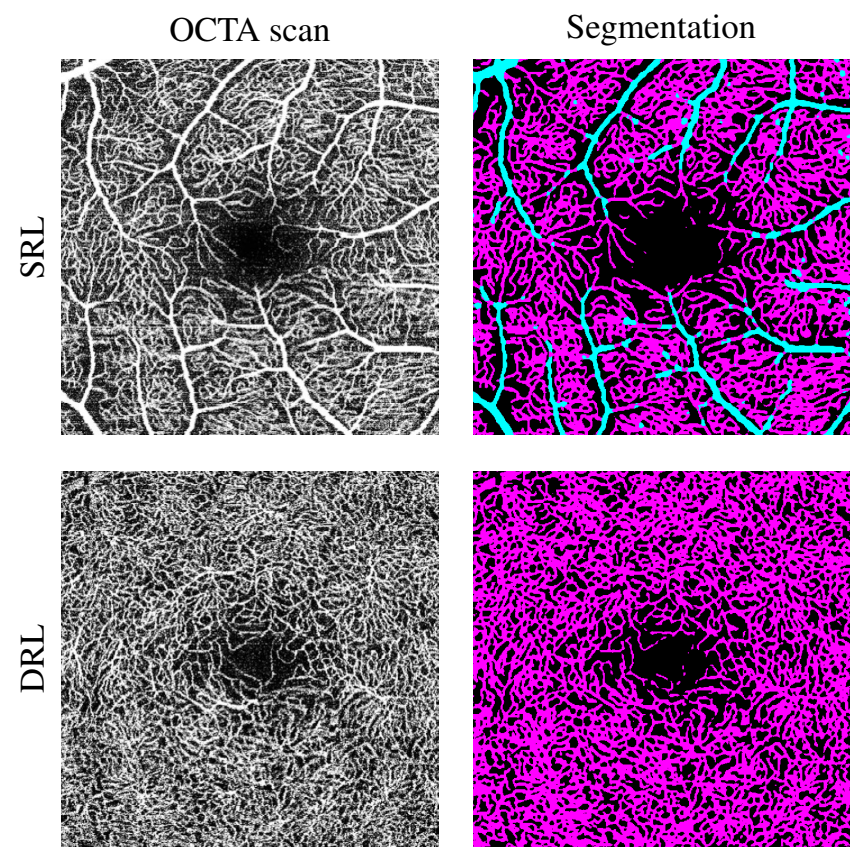

Fig. 1. An OCTA scan of size $1004 \times 1007$ pixels in the superficial and deep retinal layer along with the corresponding segmentation of the capillaries (pink) and larger vessels (blue).

Table 1. The blood values (thrombocytes (TC) and leukocytes (LC) in $10^{9} / \mathrm{L}$ and hemoglobin $(\mathrm{HG})$ in $\mathrm{mmol} / \mathrm{L}$ ) of the patients. The normal values for HG are $8.3-10.5 \mathrm{mmol} / \mathrm{L}$, for TC $145-390 \times 10^{9} / \mathrm{L}$, and for LC $3.5-8.8 \times 10^{9} / \mathrm{L}$. Green indicates normal values, red indicates abnormally high values, and blue abnormally low values.

\begin{tabular}{l|ccc|ccc|cc} 
& \multicolumn{3}{|c|}{ Patient A } & \multicolumn{3}{c|}{ Patient B } & \multicolumn{2}{c}{ Patient C } \\
Scan & 1 & 2 & 3 & 1 & 2 & 3 & 1 & 2 \\
\hline TC & 749 & 502 & 365 & 186 & 341 & 314 & 660 & 279 \\
LC & 5.5 & 6.0 & 2.3 & 2.7 & 4.0 & 4.2 & 8.9 & 2.8 \\
HG & 9.0 & 8.6 & 8.5 & 10.8 & 8.8 & 9.3 & 12.8 & 8.0 \\
\hline
\end{tabular}

jects, to investigate the uncertainty of the approach, and to patients with hematological disorders before and after cytoreductive medical treatment where we expect vascular changes. Due to the normalization of the blood values after treatment, we assume that there will be physiological changes present either in blood flow, the capillary density, or in the thickness of the capillaries. In this work, we look at the changes in the local capillary density, which we assume also reflect both the capillary thickness and the number of perfused capillaries above the lower limit of detection.

\subsection{Data and scanning protocol}

The study includes eight participants of which three are diagnosed with hematological diseases (patients A, B, and C with average age of $59.3 \pm 7.6$ ) and five are healthy controls (average age $47.4 \pm 19.1$ ). Patient A has essential thrombocytosis, while patients $\mathrm{B}$ and $\mathrm{C}$ have polycythaemia vera, as 
Table 2. Global capillary densities (a unit-less ratio ranging from 0 to 1 ) of the nine healthy control (HC) eyes and the patients' eyes before (scan 1), during and after treatment (scan 2 and 3) in the superficial and deep retinal layers. $\mathrm{R}$ is right eye and $\mathrm{L}$ is left eye.

\begin{tabular}{cc|cc|cc|cc|cc|c||cc|cc|c} 
& & \multicolumn{2}{|c}{ HC 1 } & \multicolumn{2}{c}{ HC 2 } & \multicolumn{2}{c|}{ HC 3 } & \multicolumn{2}{c|}{ HC 4 } & HC 5 & Patient A & Patient B & Patient C \\
& & $\mathrm{R}$ & $\mathrm{L}$ & $\mathrm{R}$ & $\mathrm{L}$ & $\mathrm{R}$ & $\mathrm{L}$ & $\mathrm{R}$ & $\mathrm{L}$ & $\mathrm{R}$ & $\mathrm{R}$ & $\mathrm{L}$ & $\mathrm{L}$ & $\mathrm{R}$ & $\mathrm{L}$ \\
\hline \multirow{3}{*}{ SRL } & Scan 1 & 0.40 & 0.39 & 0.37 & 0.45 & 0.36 & 0.32 & 0.39 & 0.37 & 0.31 & - & 0.42 & 0.38 & 0.28 & 0.31 \\
& Scan 2 & 0.40 & 0.38 & 0.39 & 0.42 & 0.36 & 0.31 & 0.39 & 0.39 & 0.32 & 0.42 & 0.41 & 0.35 & 0.29 & 0.32 \\
& Scan 3 & - & - & - & - & - & - & - & - & - & 0.39 & - & 0.38 & - & - \\
\hline \multirow{2}{*}{ DRL } & Scan 1 & 0.54 & 0.54 & 0.58 & 0.58 & 0.55 & 0.55 & 0.49 & 0.51 & 0.44 & - & 0.53 & 0.46 & 0.41 & 0.40 \\
& Scan 2 & 0.55 & 0.56 & 0.58 & 0.58 & 0.53 & 0.55 & 0.47 & 0.50 & 0.45 & 0.49 & 0.53 & 0.47 & 0.45 & 0.47 \\
& Scan 3 & - & - & - & - & - & - & - & - & - & 0.55 & - & 0.45 & - & -
\end{tabular}

can be seen in Table 1. Both eyes were scanned in all patients aside two; in patient $\mathrm{B}$ and in one control, only one eye was scanned. Patients were examined at least twice: A baseline at the time of diagnosis (scan 1), and one or two follow-up examination after treatment (scan 2 and 3). The healthy controls were examined at two different times of short interval. Examinations were approved by the Institutional Review Board and included measurement of best-corrected visual acuity and dilated pupil OCTA using Spectralis Heidelberg Engineering, Heidelberg, Germany. For pupil dilation participants received one drop of tropicamide $1.0 \%$. A macula centered OCTA was performed giving approximately $3 \times 3 \mathrm{~mm}^{2}$ field of view. Because of the high correlation between vessel density and signal strength [14] we use only well-focused scans with little motion artefacts and with a signal strength of more than 35 $\mathrm{dB}$ in all scan sessions.

\subsection{Statistical analysis}

For each local density change map, the distribution of values is collected, and its mean and standard deviation are computed. The one-sample Kolmogorov-Smirnov test is used to compare these distributions to a normal distribution with the same mean and standard deviation. Here, the test statistic $K$ is the maximum absolute difference between the normal distribution and the empirical cumulative distribution of the data.

\section{RESULTS AND DISCUSSION}

Fig. 1 shows the segmentation output of one of the healthy subjects. For all subjects, the segmentation of the OCTA images looks correct after visual inspection. This shows that the dictionary segmentation model is applicable to images from another OCTA platform, since it has been created for OCTA images acquired by a swept source-OCT from Topcon Medical Systems, Inc., while we in this work use data acquired by Spectralis Heidelberg Engineering.

The blood values of the patients can be seen in Table 1 . The thrombocyte-values in patient A are normalized after the cytoreductive medical treatment, although the leukocytes are abnormally low at time of scan 3. All blood values are completely normal for patient $\mathrm{B}$ at scan 2 and 3, and for patient $\mathrm{C}$ at scan 2 the leukocyte-value is abnormally low. The leukocytes are large and have a tendency to stick together, and hence a higher vascular change is expected for patient $C$.

The global capillary densities are described in Table 2. They show few changes for the controls which indicate the uncertainty of the method. There are also few changes for the patients in the SRL, although, an increase is observed for patient $\mathrm{A}$ and $\mathrm{C}$ in the DRL after treatment.

Fig. 2 shows two examples of the local density change and their distributions - one for a healthy control and one for patient $\mathrm{C}$. Once again, the density change for the healthy control indicates the amount of uncertainty in the results due to the acquisition noise and the limitations of the method. It is seen that the local density change between the two scans for the healthy subject is more normally distributed and centered around zero. For patient $\mathrm{C}$, there seems to be an increase in the local capillary density after treatment, and the increase is larger than the noise shown in the healthy subject. Despite the overall increase, there is a small local decrease in the capillary density in patient $\mathrm{C}$, which could be due to an artefact.

Fig. 3 shows the relationship between $K$, the mean and the standard deviation (STD) of the distribution of the density changes. We see that the distributions for the healthy subjects have low standard deviations as well as relatively low $K$-values indicating that they are normally distributed, which is as expected since they are caused by the acquisition noise. Their means are also relatively low. When observing the mean, the values of the DRL and SRL corresponding to the same patient are sometimes mirrored around zero. This could be a sign of faulty detection of the boundary between DRL and SRL. The hematologic patients are characterized by larger standard deviations and sometimes larger $K$-values. Note that all blood values are normal in scan 2 and scan 3 for patient $\mathrm{B}$, and the values of the density change between these two scans are closer to the controls. Based on the blood values, we expect the largest vascular changes for patient $\mathrm{C}$, and these distributions also deviate more from a normal distribution based on the $K$-value. We also see a large increase in the capillary density in the DRL for both eyes based on the mean. It should be noted that if the blood flows too slowly, it cannot be detected by the OCTA device. Hence, it could seem like the blood flows faster or the capillaries are more dilated in DRL after the medical treatment. 

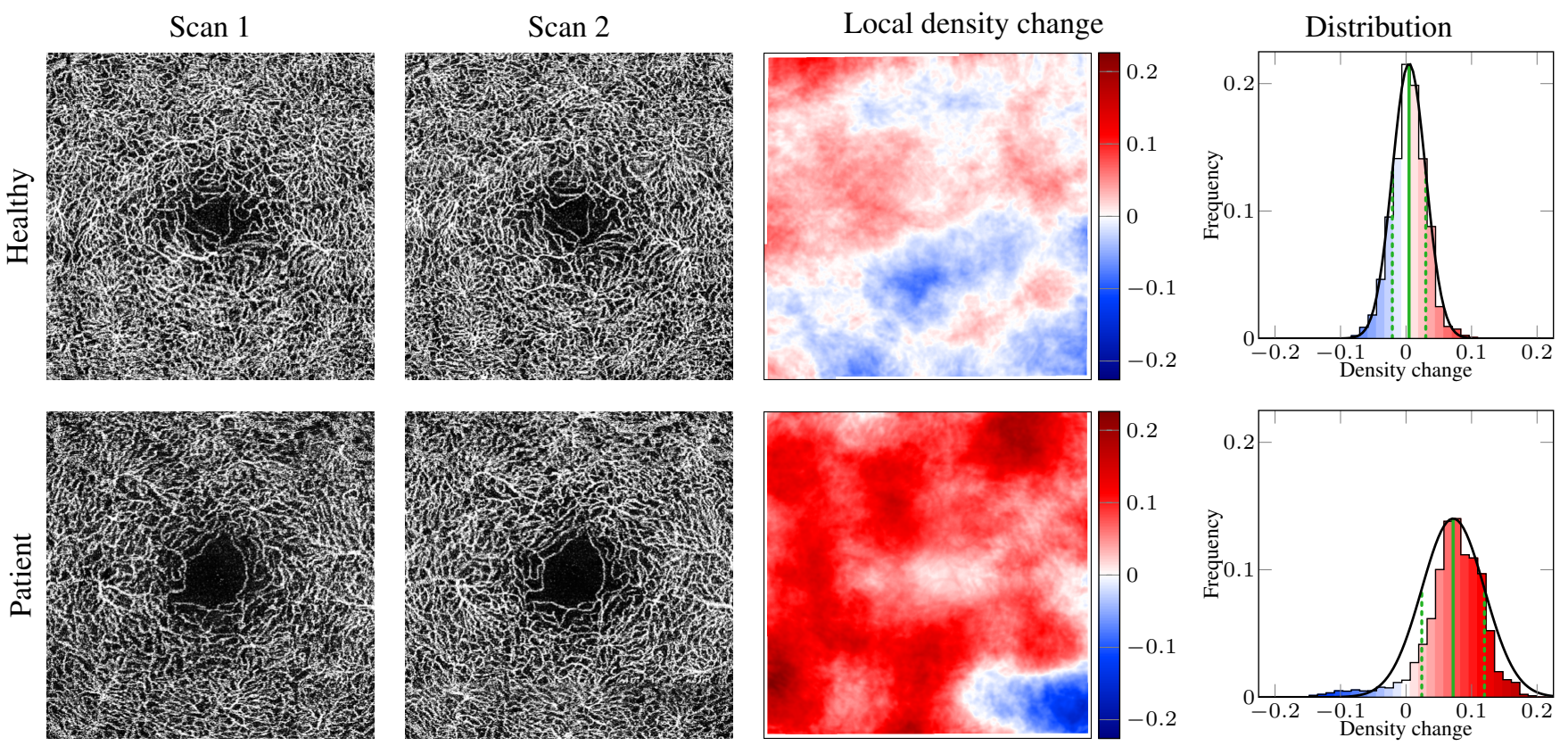

Fig. 2. Top row: Deep layer of a healthy subject. Bottom row: Deep layer of patient C. Two columns on the left: OCTA scans. Third column: The local density change, where red indicates an increase in the local signal compared to the previous scan, while blue indicates a decrease. Rightmost column: The corresponding distributions of the density change with the mean (green) and standard deviation (dashed green).
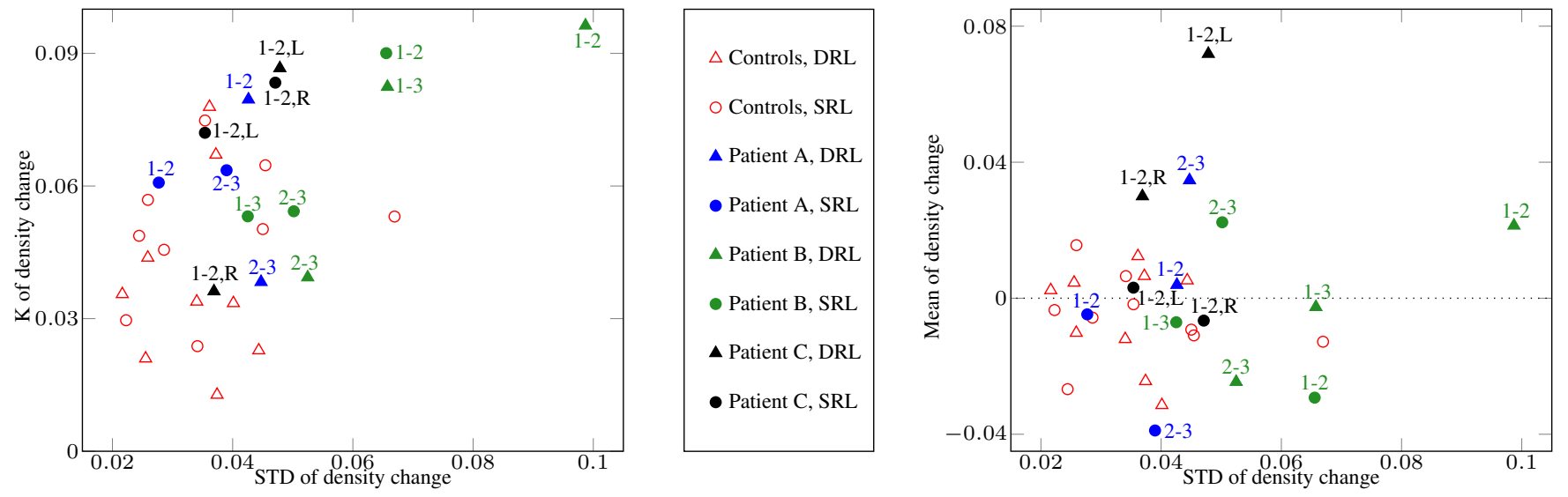

Fig. 3. The relationship between test statistics $K$, the mean and the standard deviation (STD) of the density change for each subject. The marker shape indicates whether it is the superficial retinal layer (circle) or deep retinal layer (triangle). For patients, the two scans that are compared are written next to the marker. For patient C, L and R indicate the left and right eye, respectively.

Although the results of this study indicate that the vascular changes of the patients are different from the controls, the sample size is relatively small, and more data would be needed to draw firm conclusions. Additionally, potential structural changes can be blurred out by the smoothing effect of the local density estimation. It would be interesting to study potential changes in the capillary thickness, although this is challenging to do with the current resolution of OCTA images. Optimally, the 3D vasculature should be analysed and quantified, but raw data from the scanners can be difficult to obtain, and hence this study has only used en face angiograms.

\section{CONCLUSION}

Our investigation indicates that OCTA data combined with an automated segmentation method can be used for measuring changes in capillary densities. The study was carried out on only three hematologic patients undergoing treatment, but these had generally larger changes than healthy controls, despite the relatively large uncertainty seen in the control subjects. This shows promise in extending the method, and can potentially provide insight into vascular effects of hematologic disorders given a larger sample size. 


\section{REFERENCES}

[1] Anne Willerslev et al., "Non-invasive imaging of retinal blood flow in myeloproliferative neoplasms," Acta Ophthalmol, vol. 95, no. 2, pp. 146-152, 2017.

[2] A Yasin Alibhai et al., "Quantifying microvascular changes using oct angiography in diabetic eyes without clinical evidence of retinopathy," Ophthalmology Retina, vol. 2, no. 5, pp. 418-427, 2018.

[3] Zhongdi Chu et al., "Quantitative assessment of the retinal microvasculature using optical coherence tomography angiography," J Biomed Opt, vol. 21, no. 6, pp. 066008, 2016.

[4] Nicholas A Iafe et al., "Retinal capillary density and foveal avascular zone area are age-dependent: quantitative analysis using optical coherence tomography angiography," Invest Ophthalmol Vis Sci, vol. 57, no. 13, pp. 5780-5787, 2016.

[5] Nabila Eladawi et al., "Automatic blood vessels segmentation based on different retinal maps from octa scans," Computers in biology and medicine, vol. 89, pp. 150-161, 2017.

[6] Santosh GK Gadde et al., "Quantification of vessel density in retinal optical coherence tomography angiography images using local fractal dimension," Invest Ophthalmol Vis Sci, vol. 57, no. 1, pp. 246-252, 2016.

[7] Mayss Al-Sheikh et al., "Repeatability of automated vessel density measurements using optical coherence tomography angiography," Br J Ophthalmol, vol. 101, no. 4, pp. 449-452, 2017.

[8] Jianqin Lei et al., "Repeatability and reproducibility of superficial macular retinal vessel density measurements using optical coherence tomography angiography en face images," JAMA ophthalmology, vol. 135, no. 10, pp. 1092-1098, 2017.

[9] Lisa Toto et al., "Association between outer retinal alterations and microvascular changes in intermediate stage age-related macular degeneration: an optical coherence tomography angiography study," British Journal of Ophthalmology, vol. 101, no. 6, pp. 774-779, 2017.

[10] Changlei Dongye et al., "Automated detection of dilated capillaries on optical coherence tomography angiography," Biomed Opt Express, vol. 8, no. 2, pp. 1101-1109, 2017.

[11] Ahmed M Hagag et al., "OCT angiography changes in the 3 parafoveal retinal plexuses in response to hyperoxia," Ophthalmol Retina, vol. 2, no. 4, pp. 329-336, 2018.

[12] Minjie Chen, Jinhui Dai, and Lan Gong, “Changes in retinal vasculature and thickness after small incision lenticule extraction with optical coherence tomography angiography," J Ophthalmol, vol. 2019, 2019.

[13] Asli Inal et al., "Optical coherence tomography angiography: Are there any changes in measurements after strabismus surgery?," J Pediatr Ophthalmol Strabismus, vol. 56, no. 2, pp. 95-100, 2019.

[14] J Yu Jeffrey et al., "Signal strength reduction effects in optical coherence tomographic angiography," Ophthalmol Retina, 2019.

[15] Astrid M E Engberg et al., "Automated quantification of retinal microvasculature from OCT angiography using dictionary-based vessel segmentation," in Communications in Computer and Information Science. Springer, 2019.

[16] Anders B Dahl and Vedrana A Dahl, "Dictionary based image segmentation," in Image Analysis. 2015, pp. 2637, Springer International Publishing.

[17] David G Lowe, "Distinctive image features from scaleinvariant keypoints," Int J Comput Vis, vol. 60, no. 2, pp. 91-110, 2004.

[18] Andrea Vedaldi and Brian Fulkerson, "VLFeat: An open and portable library of computer vision algorithms," http://www.vlfeat.org/, 2008. 